\title{
Plataforma Inteligente e Sustentável para Coleta de Resíduos baseada em IoT e LPWAN
}

\author{
Lahis G. de Almeida ${ }^{1}$, Juliana F. Borin ${ }^{1}$ \\ ${ }^{1}$ Instituto de Computação - Universidade Estadual de Campinas (Unicamp) \\ Campinas - SP - Brasil \\ lahis.almeida@students.ic.unicamp.br, juliana@ic.unicamp.br
}

\begin{abstract}
One of the challenges in Smart Cities is Waste Collection; traditionally done through routes that do not guarantee waste collectors will be able (full) for collection, it may result in the misspend of resources such as fuel, generating consequent increase in the emission of polluting gases. This paper proposes a smart and sustainable platform for waste collection, based on Internet of Things, responsible for monitoring the filling level of collectors, generating optimized collection routes, and sustainability indicators. The results presented in this paper refer to collectors level monitoring stage.
\end{abstract}

Resumo. Um dos desafios no contexto de Cidades Inteligentes está na Coleta de Resíduos; feita tradicionalmente por meio de rotas que não garantem que os coletores de resíduos estarão aptos (cheios) para a coleta, esta atividade pode resultar em desperdício de recursos como combustível e gerar consequente aumento na emissão de gases poluentes. Este artigo propõe uma plataforma inteligente e sustentável para coleta de resíduos, baseada em Internet das Coisas, responsável por monitorar o nível de enchimento dos coletores, gerar rotas de coleta otimizadas e indicadores de sustentabilidade. Os resultados apresentados referem-se à etapa de monitoramento do nível de enchimento dos coletores.

\section{Introdução}

Cidades Inteligentes (Smart Cities) podem ser definidas como sistemas nos quais os cidadãos, por meio de tecnologias de informação e comunicação, podem interagir e utilizar os ativos da cidade, estimulando o desenvolvimento econômico e melhora na qualidade de vida da população [Shyam et al. 2017]. Seus ativos constituem, de forma geral, sistemas de informação e comunicação, bibliotecas, escolas, hospitais e sistemas de transporte.

O conceito de Smart Cities acompanha as pautas de desenvolvimento sustentável e econômico [Pardini et al. 2019]. Em 2015, a Organização das Nações Unidas (ONU) lançou a Agenda 2030, que reúne 17 Objetivos de Desenvolvimento Sustentável (ODS) que abrangem as 3 dimensões do desenvolvimento sustentável - social, ambiental e econômica - e podem ser colocados em prática por governos, sociedade civil e setor privado [ECAM 2021]. Entre as aplicações existentes para Smart Cities, a coleta inteligente de resíduos possui impacto significativo no meio ambiente e qualidade de vida dos cidadãos [Pardini et al. 2019]. A geração de resíduos vem se tornando uma questão crítica para países com crescimento populacional elevado. Em 2012 foi previsto que poderia aumentar em 2.2 bilhões de toneladas até 2025, indicando que a geração de resíduos sólidos urbanos (RSU), está crescendo de forma mais acentuada que a taxa de urbanização, que 
deve chegar a $66 \%$ até 2050 . Isso faz da coleta de resíduos um dos principais desafios das cidades, já que o descarte inadequado e problemas como transbordamento nos coletores podem prejudicar gravemente o meio ambiente e contribuir para a disseminação de doenças que afetam a saúde pública [Sohag and Podder 2020].

No modelo tradicional de coleta de resíduos, as rotas de coleta são estáticas, ou seja, os caminhões de coleta seguem rotas previamente planejadas, desconsiderando o fato dos coletores de resíduos (lixeiras) se encontrarem realmente cheios ou não [Pardini et al. 2019]. Entretanto, em determinadas regiões, viagens de um local a outro requererem um deslocamento considerável e saber previamente se os coletores do local de destino estão cheios pode significar economia de combustível e tempo. A cidade de São Petersburgo, Rússia, evidencia a importância da coleta eficiente de resíduos [Anagnostopoulos et al. 2017]. Em 2017, a sua média de resíduos sólidos produzidos era de 1,7 milhão toneladas/ano. Diariamente, o município utiliza 476 caminhões de coleta, que seguem rotas estáticas, com capacidade de 5 T/caminhão. A quantidade de combustível consumido em um ano é de 1,8 milhão de litros o que gera um custo anual de mais de 1 milhão de dólares.

O desenvolvimento de aplicações para melhorar a coleta de resíduos é estimulado por iniciativas públicas destinadas a criar ambientes sustentáveis, que reduzam as emissões de gases poluentes decorrentes da coleta, e por regulações mais rígidas para o fim do descarte ilegal. O avanço de tecnologias baseadas em Internet das Coisas (IoT) é outro fator que impulsiona o desenvolvimento dessas soluções [Sheng et al. 2020]. Diferente do cenário de coleta tradicional, a Coleta Inteligente de Resíduos abrange o monitoramento de coletores e planejamento da rota de coleta [Fedchenkov et al. 2017]. Por meio de tecnologias IoT, esses sistemas inteligentes proveem a interação entre os diferentes componentes dessa gestão, desde o sensoriamento dos coletores, caminhões de coleta até o destino final (fábricas de reciclagem e lixões). Os coletores desse tipo de sistema, comumente contém sensores que informam o estado do recipiente (e.g., nível de enchimento, temperatura, umidade). Um fator importante para determinar a eficácia de sistemas de Coleta Inteligente de Resíduos, consiste no alcance da infraestrutura que provê comunicação para os pontos de coleta [Sheng et al. 2020]. Tecnologias de comunicação como LoRa e SigFox, que operam em uma rede de longa distância e baixa potência (LPWAN), são capazes de atender aos requisitos de comunicação necessários para estes tipos de sistema.

Neste contexto de Smart Cities, a Prefeitura Universitária da Unicamp desenvolveu a iniciativa Smart Campus ${ }^{1}$, onde o conceito de IoT é explorado na gestão mais eficiente do campus por meio da coleta de informações provindas de objetos inteligentes. Diversos projetos em prol da comunidade universitária estão sendo implementados em áreas como segurança, transporte e meio ambiente. Entre eles, o projeto da Coleta Diferenciada tem como objetivo o planejamento de rotas otimizadas para a coleta dos resíduos do tipo pilhas e baterias [Hatta and Borin 2018]. Para isto, uma infraestrutura IoT piloto foi desenvolvida, entretanto, em sua fase atual, o projeto apresenta desafios como eficiência energética, sensoriamento e tecnologias de comunicação. Em vista desses desafios, este trabalho tem como principais contribuições: (i) propor uma plataforma inteligente e sustentável para a coleta de resíduos, baseada em IoT e LPWAN; (ii) avaliar a primeira etapa desta plataforma, que consiste no monitoramento do nível de enchimento dos coletores inteligentes; e (iii) definir indicadores de sustentabilidade relacionados à co-

\footnotetext{
${ }^{1}$ http://smartcampus.prefeitura.unicamp.br/
} 
leta de resíduos e baseados nos ODSs que possam ser gerados pela plataforma para tornar a Cidade Universitária da Unicamp mais sustentável.

O restante do trabalho está organizado da seguinte forma: a Seção 2 apresenta os trabalhos relacionados com a coleta de resíduos e cases de coletores inteligentes, a Seção 3 descreve o projeto de coleta de pilhas e baterias do Smart Campus Unicamp, a Seção 4 descreve a abordagem proposta, a Seção 5 apresenta os experimentos realizados no monitoramento de nível dos coletores, a Seção 6 apresenta a discussão dos resultados obtidos; e, por fim, a Seção 7 apresenta as conclusões e trabalhos futuros.

\section{Trabalhos Relacionados}

Diversos trabalhos na literatura relatam o uso das tecnologias IoT na coleta de resíduos [Pardini et al. 2019]. Cruz et al. [2021], estudam o uso da tecnologia LPWAN na coleta de resíduos para a Smart City de Lisboa. As tecnologias LoRa (Long Range) e LoRAWAN (LoRa Wide Area Network) foram escolhidas e os resultados positivos serviram para promover a implantação de uma rede $L o R a$ em toda a cidade.

O sistema inteligente proposto por Anh Khoa et al. [2020], utilizou LoRaWAN, sensores ultrassônicos e algoritmos de roteamento para otimizar a coleta de resíduos na Universidade Ton Duc Thang (Vietnã), resultando em economia de custo, mão de obra e tempo de serviço. Melakessou et al. [2020] apresentaram uma plataforma de gerenciamento de resíduos para empresas. O sistema se baseou na LPWAN Sigfox, sensores ultrassônicos e em técnicas de aprendizado de máquina, mostrando que os caminhos de coleta poderiam ser otimizados minimizando a distância total percorrida e o tempo de serviço no local com base nos perfis de cada cliente.

Baldo et al. [2021] e Marques et al. [2019] propuseram arquiteturas de rede para solucionar o problema da coleta tradicional de resíduos. Baldo et al. utilizou uma arquitetura baseada em LoRaWAN, que foi capaz de realizar a coleta de forma eficaz, além de detectar incêndios em materiais residuais e realizar previsão da geração futura de resíduos. Marques et al., por sua vez, utilizaram uma arquitetura baseada em WiFi, que foi capaz de gerenciar até 3.902 lixeiras simultaneamente.

Bakhshi and Ahmed [2018] e Lozano et al. [2018] também propuseram soluções IoT para a coleta de resíduos. Bakhshi and Ahmed utilizaram em sua arquitetura de rede a tecnologia WiFi e, como resultados, obtiveram economia de combustível de até $46 \%$ e redução do tempo de coleta de até $18 \%$. A solução de Lozano et al., por sua vez, baseou-se em rotas dinâmicas e na tecnologia LoRaWAN, tendo como resultados de suas simulações grande cobertura alcançada com implantações mínimas de antenas na região (9 gateways LoRa) e o sistema de otimização de rotas, garantiu economia de $28 \%$ nas distâncias percorridas, proporcionando economia de combustível e tempo em comparação com a abordagem antiga de coleta com rota estática.

Em relação à soluções IoT para a coleta de resíduos que evidenciam suas contribuições para melhor desenvolvimento sustentável nas Smart Cities, pode-se citar as pesquisas de Hussain et al. [2020], Fatimah et al. [2020] e Ali et al. [2020]. Hussain et al. destacaram a importância da concentração de poluentes atmosféricos como fator a ser levado em consideração na coleta de resíduos. Dessa forma, além de gerenciar o descarte de mesmos, seu trabalho também prevê, por meio de técnicas de aprendizado de 
máquina, a quantidade do poluente atmosférico $(\mathrm{CO})$ presente no ambiente circundante do coletor, permitindo tomada de ação corretiva com a coleta antecipada.

Fatimah et al. [2020] propuseram o estudo de um sistema inteligente e sustentável que pode alcançar bons desempenhos econômicos, sociais e ambientais na gestão de resíduos, apresentando processos de economia circular para separar os resíduos municipais, identificar as características dos resíduos e determinar tecnologias de tratamento dos mesmos por meio do uso de tecnologias IoT como integradoras. Ali et al. [2020] descreveram um sistema para a cidade de Najran capaz de realizar a coleta inteligente, detectar incêndios em materiais residuais e prever a geração futura de resíduos, com o objetivo de evitar o transbordamento dos coletores e reduzir o efeito da taxa de poluição no meio ambiente em relação aos métodos tradicionais de coleta.

Tabela 1. Comparativo entre trabalhos relacionados e solução proposta.

\begin{tabular}{|c|c|c|c|c|c|c|c|c|}
\hline Trabalho & Resíduo & Sensor de nível & AD & Placa & Rede & Ambiente & RD & IND \\
\hline Cruz et al. [2021] & sólido & US (DF702) & $x$ & TTGO ESP32 & LoRaWAN & Real & $x$ & $x$ \\
\hline Baldo et al. [2021] & sólido & US (JSN-SR04T) & $\checkmark$ & ATtiny84 & LoRaWAN & Simulado/Real & $x$ & $x$ \\
\hline Hussain et al. [2020] & sólido & US (HC-SR04) & $x$ & Arduino Uno & $W i F i$ & Real & $\checkmark$ & $\checkmark$ \\
\hline Melakessou et al. [2020] & sólido & US (Brighterbins) & $\checkmark$ & $x$ & Sigfox & Real & $\checkmark$ & $x$ \\
\hline Anh Khoa et al. [2020] & sólido & US (HC-SR04) & $x$ & ATmega328 & LoRaWAN & Simulado/Real & $\checkmark$ & $x$ \\
\hline Ali et al. [2020] & sólido & US (HC-SR04) & $x$ & ATmega328 & $W i F i$ & Simulado & $\checkmark$ & $\checkmark$ \\
\hline Marques et al. [2019] & sólido/orgânico & $x$ & $x$ & NodeMCU & WiFi & Real & $x$ & $x$ \\
\hline Bakhshi and Ahmed [2018] & sólido & US (HC-SR04) & $x$ & Raspberry Pi & $W i F i$ & Real & $\checkmark$ & $x$ \\
\hline Lozano et al. [2018] & sólido/orgânico & US (JSN-SR04T) & $x$ & SAML21 & LoRaWAN & Simulado & $x$ & $x$ \\
\hline Solução proposta & sólido & LIDAR (VL53L0X) & $\checkmark$ & Heltec ESP32 & LoRaWAN & Simulado/Real & $\checkmark$ & $\checkmark$ \\
\hline
\end{tabular}

A Tabela 1 compara os componentes principais utilizados pelos trabalhos descritos nesta seção e a solução proposta. Praticamente todos os trabalhos utilizam um sensor ultrassônico (US) para medir o nível de coletores de resíduos sólidos. Entretanto, apenas Baldo et al. e Melakessou et al. apresentaram análise de desempenho (AD) destes sensores. Em relação ao custo, os sensores US DF702 ( $\left.R \$ 422,46^{2}\right)$, JSN-SR04T $\left(R \$ 34,62^{3}\right)$ e Brighterbins ( $R \$ 1.119,58^{4}$ ) não são acessíveis para soluções em larga escala, contrariando os objetivos de baixo custo desta pesquisa. Outro desafio observado, é que apesar de todos os trabalhos terem como objetivo direto ou indireto substituir a rota estática pela rota dinâmica (RD) a partir do monitoramento de nível feito pelos coletores inteligentes, são poucos os trabalhos que implementam esta etapa de fato. Em relação às contribuições relacionadas ao desenvolvimento sustentável nas Smart Cities, apesar dos trabalhos de Hussain et al. e Ali et al. medirem os níveis de $\mathrm{CO}$ e taxa de poluição, eles não utilizam nenhum tipo de indicador (IND) para medir a quantidade de resíduo gerado por região. Fatimah et al. por sua vez, relaciona a sua solução com os ODS, mas não entra em detalhes quantitativos de como medirá tal contribuição.

Portanto, como diferenciais em relação aos demais trabalhos esta pesquisa, que está em fase inicial, utiliza o sensor LIDAR (VL53L0X) para medir o nível dos coletores

\footnotetext{
$2_{\text {https: //bit.ly/us-df702 }}$

3 https://bit.ly/us-jsn-sr04t

$4_{\text {https }}$ //bit.ly/us-brighterbins
} 
de resíduos sólidos, definido após estudo comparativo, de desempenho e custo, com o sensor US (HC-SR04). Além de apresentar desempenho superior ao US (como será mostrado na Seção 6), o LIDAR possui modos de operação com baixo consumo energético, importante para garantir a sustentabilidade da solução. Este trabalho também é proposto uma plataforma completa de Coleta de Resíduos, ou seja, desde os coletores inteligentes à interface com os gestores e utilização de rotas dinâmicas; adicionalmente, serão produzidos indicadores de sustentabilidade relacionados à emissão de gases poluentes e geração de resíduos em fases futuras de implementação da plataforma, possibilitando mensurar e detalhar como os resultados que serão obtidos contribuirão com os ODSs.

\section{Coleta Inteligente no Smart Campus Unicamp}

A Unicamp possui 47 coletores para pilhas e baterias espalhados pelo campus de Barão Geraldo [Prefeitura Universitária 2016]. Entretanto, atualmente, a cada coleta, os motoristas dos caminhões trafegam pelo campus inteiro, seguindo uma rota estática que pode levar em direção a coletores vazios ou com nível de preenchimento baixo, tornando a coleta desnecessária nestes casos. Para reverter este cenário, o projeto de Coleta Diferenciada do Smart Campus foi criado [Hatta and Borin 2018]. Em sua arquitetura, sensores medem o nível de enchimento dos coletores e enviam os dados, via WiFi, para uma plataforma de IoT na nuvem (Konker ${ }^{5}$ ) utilizando o protocolo de comunicação MQTT. A interface com os usuários se dá por meio de um sistema web que, quando acessado, envia uma requisição HTTP para a plataforma IoT com o objetivo de obter as informações de cada coletor. Dessa forma, os usuários do sistema, ou seja, funcionários responsáveis pela coleta de pilhas e baterias, podem consultar o nível de enchimento dos coletores (\%), definir pontos de partida e chegada do caminhão de coleta e, com essas informações, gerar a rota otimizada para que os veículos realizem a coleta desses resíduos.

Após validação desta arquitetura pela Prefeitura Universitária, notou-se que o sensor utilizado no monitoramento de nível do coletor apresenta instabilidade nas leituras de distância ao longo do tempo, ou seja, apresenta frequente variação entre leituras corretas e incorretas, prejudicando a confiabilidade da solução final. Em relação à eficiência enérgica, o hardware da solução consome $18 \mathrm{~mA}$, necessitando que o power bank utilizado para alimentar o protótipo tenha que ser carregado a cada 10 dias, demandando mão de obra extra para essa troca [Hatta and Borin 2018]. Já no envio dos dados para a nuvem, a tecnologia WiFi não é a ideal pois seu alcance é limitado e muitas vezes não está presente nos locais onde estão os coletores. Adicionalmente, a quantidade de dados transmitida por essa aplicação é pequena, não necessitando da quantidade elevada de banda proporcionada por essa tecnologia. Portanto, este projeto apresenta desafios referentes, à medição do sensor, eficiência energética e a comunicação entre coletor e nuvem.

\section{Abordagem Proposta}

Este trabalho propõe uma plataforma inteligente para coleta de resíduos, baseada em IoT e LPWAN, propondo soluções para os desafios presentes no projeto Coleta Diferenciada e geração de indicadores de sustentabilidade. Em relação a desafio de monitoramento de nível, serão realizados testes e análises comparativas dos sensores mais apropriados para esta tarefa. Em relação aos desafios de consumo energético e infraestrutura de rede, será

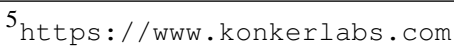


proposta a utilização das tecnologias LPWAN LoRa, bem como o protocolo LoRaWAN, que define a arquitetura de rede e seus parâmetros de comunicação [Pereira 2017], possibilitando satisfazer os requisitos de longa distância e baixo consumo energético necessários para eficácia da Coleta Inteligente de Resíduos.

$\mathrm{Na}$ arquitetura proposta para a plataforma (Figura 1), os nós sensores da infraestrutura de rede são os coletores inteligentes (I). Eles enviarão, via protocolo LoraWAN, as informações de identificação (ID) e capacidade (\%) para o Gateway LoRa (II). Este receberá as informações e as enviará, via MQTT, para o Network Server gerenciá-las (e.g., tratar colisões, pacotes duplicados) (II). O Network Server enviará as informações para a plataforma de nuvem (IV), que as encaminhará para o sistema web, via HTTP (V). Por fim, a cada acesso ao sistema web, é possível obter os dados dos coletores armazenados na nuvem, possibilitando a consulta da porcentagem do nível de enchimento, identificação e localização do coletor; permitindo a definição de pontos de partida/chegada dos veículos para a geração da rota otimizada para a coleta dos resíduos (VI).

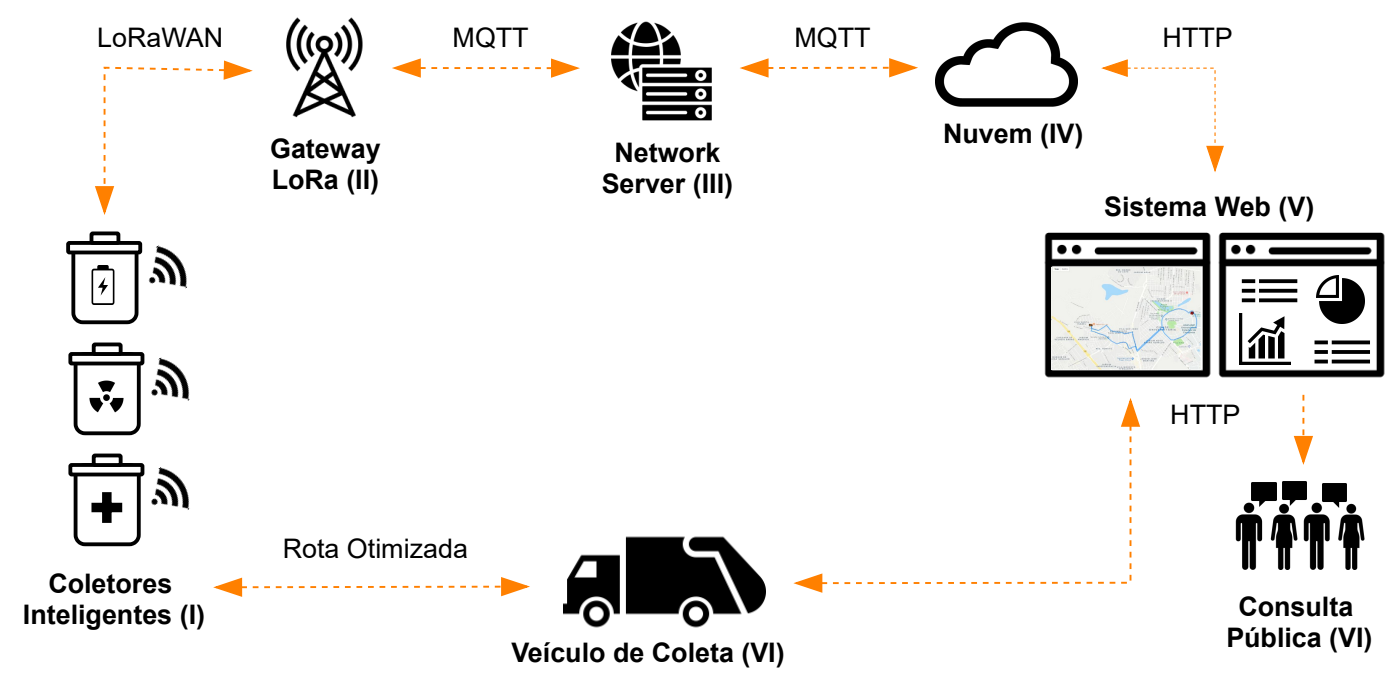

Figura 1. Arquitetura da Plataforma Inteligente para Coleta de Resíduos.

Por meio do coletor inteligente, é possível saber a quantidade de resíduos descartados e a quantidade de emissão de gases como $\mathrm{CO}$ e $\mathrm{CO} 2$, que estão diretamente relacionados ao consumo de combustível demandando pelas rotas de coleta de resíduos. A partir destes dados, a plataforma proposta poderá contribuir com os ODS 11, 12 e 13 estipulados pela ONU, referentes à cidades sustentáveis, consumo e produção responsáveis, e ação contra mudanças climáticas, respectivamente [SDSN 2021]. Os indicadores que podem ser produzidos por esta solução são apresentados na Tabela 2.

O indicador 71 é referente à porcentagem de resíduos sólidos urbanos coletados e bem gerenciados e os demais indicadores são relacionados à poluição urbana. A partir da implementação desses indicadores no sistema web, que possibilitará consulta pública dos mesmos, será possível, p. ex., traçar comparativos entre a emissão de carbono antes e depois de aplicada a solução, além de informar a quantidade de descarte por semana, mês ou ano de cada unidade da Unicamp, contribuindo para a criação de ações de planejamento de metas anuais de redução de carbono e descarte adequado de resíduos no campus. 
Tabela 2. Indicadores de sustentabilidade produzidos pela Plataforma.

\begin{tabular}{ccc}
\hline ODS & Indicadores & Descrição \\
\hline \hline $\mathbf{1 1}$ e 12 & 69 & Poluição média do ar urbano por material particulado. \\
\hline $\mathbf{1 1}$ e 12 & 71 & Porcentagem de RSUs regularmente coletados e bem gerenciados. \\
\hline $\mathbf{1 2}$ & 74 & Consumo de substâncias destruidoras da camada de ozônio. \\
\hline $\mathbf{1 3}$ & 77 & Implementação de estratégia de descarbonização. \\
\hline $\mathbf{1 3}$ & 79 & Emissões líquidas de gases do Efeito Estufa. \\
\hline
\end{tabular}

\section{Experimentos}

Os experimentos realizados neste trabalho tiveram como objetivo o monitoramento do nível do Coletor Inteligente. A seguir serão descritos o hardware e firmware final utilizados e configurações do ambiente de teste.

\subsection{Hardware e Firmware do Coletor Inteligente}

Antes de iniciar os testes de monitoramento do coletor, foram levantadas outras alternativas de sensores além do sensor ultrassônico (HC-SR04) utilizado pela Prefeitura. As melhores alternativas, considerando fatores como custo e precisão, consistiam em: sensor infravermelho (E18-D80NK), célula de peso 50kg e o sensor LIDAR (VL53L0X).

O sensor infravermelho (IR) envia às placas controladoras valores booleanos, indicando se seu feixe de luz colidiu ou não com objetos dentro da sua faixa de detecção (3 - $80 \mathrm{~cm}$ ) [Arducore 2021]. Entretanto, possui limitações como seu ajuste de alcance de detecção ser manual (potenciômetro). Dessa forma, qualquer ajuste na distância de enchimento tolerável ou descalibração, demandaria posterior manutenção. Outro fator limitante, é seu diâmetro $(\approx 1.8 \mathrm{~cm})$ [ETT 2021], que não cobre o diâmetro do coletor, demandando que mais unidades de IR sejam utilizadas, aumentando o custo da solução.

As células de peso são comumente utilizadas na construção de balanças [Straub 2019]. A partir do momento que determinado peso é aplicado, as células enviam uma tensão proporcional ao microcontrolador. Cada célula consegue medir até $50 \mathrm{Kg}$, mas a combinação de várias unidades para aumento de capacidade é possível [Straub 2019]. Entretanto, suas desvantagens consistem na necessidade de realizar calibração inicial e de usá-las em conjunto com módulos amplificadores (e.g HX7116), visto que a tensão que vem delas possui valor muito baixo e requer amplificação para ser entendida por microcontroladores [Quadros 2021], o que aumenta o custo da solução.

O sensor ultrassônico e o LIDAR utilizam o princípio do Tempo de Vôo (Time of Flight - ToF) para medir a distância entre eles e objetos [Bertoleti 2017]. O ToF consiste no cálculo da diferença de tempo entre a emissão de um sinal e seu retorno ao sensor, após ser refletido por um objeto [TeraBee 2020]. O sinal emitido/recebido pelo ultrassônico é sonoro, enquanto que para o LIDAR é o luminoso. Uma vantagem deste sensor em relação ao ultrassônico consiste em que o microcontrolador, que receberá suas medidas,

\footnotetext{
$6_{\text {http: //bit.ly/filipeflop-hx711 }}$
} 
não precisará ficar responsável por medir o tempo de retorno do sinal, o que caracteriza uma das fontes de erro na medida dos sensores ultrassônicos; o próprio LIDAR fará o registro do tempo e enviará para o microcontrolador a distância calculada [Ferreira 2020].

A Tabela 3 apresenta as principais características dos sensores considerados. Todos eles possuem alcance de leitura dentro dos limites de distância aceitáveis, pois o coletor apresenta altura total de $35 \mathrm{~cm}$. Para o sensor de peso, uma fórmula teria que ser definida para relacionar peso e distância; entretanto, como limitante há o fato de que nem sempre as baterias e pilhas possuem o mesmo tamanho e peso. A corrente de operação e tensão de alimentação, por sua vez, está dentro dos valores convencionais suportados pela principais placas controladoras encontradas no mercado (e.g, Arduino, ESP32, Raspberry $P i)$. O LIDAR apresenta vantagem em relação ao consumo energético, pois sua corrente mínima é de 3uA, situação que ocorre quando é programado no modo Software Standby, no qual realiza a medição e em seguida entra em repouso [STMicroelectronics 2016].

Tabela 3. Comparativo de sensores de nível para o Coletor Inteligente.

\begin{tabular}{cccccc}
\hline Sensor & Modelo & Alcance de Leitura & Tensão $(\mathbf{V})$ & Corrente (mA) & Custo (R\$) \\
\hline \hline Ultrassônico & HC-SR04 & $2 \mathrm{~cm}-4 \mathrm{~m}$ & 5.0 & 15.0 & 7.70 \\
\hline Infravermelho & E18-D80NK & $3-80 \mathrm{~cm}$ & 5.0 & $10.0-15.0$ & 16.50 \\
\hline Célula de Peso & $50 \mathrm{Kg}$ & $0-50 \mathrm{Kg}$ & $3.3-5.0$ & 10.0 & 17.00 \\
\hline LIDAR & VL53L0X & $3 \mathrm{~cm}-2 \mathrm{~m}$ & 5.0 & $\mathbf{3} \mathbf{u A}-\mathbf{1 9} \mathbf{~ m A}$ & 23.50 \\
\hline
\end{tabular}

Os sensores ultrassônico e LIDAR têm como vantagem em relação aos demais sensores descritos, o fato dos valores de suas leituras serem em centímetros, ao contrário do IR e da célula de peso que têm como saída valores booleanos e em gramas, respectivamente; o que garante um melhor acompanhamento do nível de enchimento do coletor, além de permitir mudanças no limite de enchimento via software, dispensando possíveis ajustes na estrutura física do coletor. Em relação ao custo, os preços foram retirados da loja online Shopee ${ }^{7}$ sendo o de menor custo o sensor ultrassônico. Portanto, levantadas as principais alternativas de sensores de nível, optou-se por testar o sensor ultrassônico, devido suas vantagens, como sua saída ser em $\mathrm{cm}$, custo e ampla utilização na literatura (conforme Tabela 1) e o LIDAR, que apesar de seu custo, fornece várias vantagens como consumo energético, custo computacional e saída em $\mathrm{cm}$.

Para o hardware de controle foi escolhida a placa Heltec ESP32 Wifi LoRa $(V 2)^{8}$, pois incorpora diversas interfaces de comunicação, entre elas o rádio Lora SX1276 ${ }^{9}$, que será utilizado na etapa de avaliação de infraestrutura de rede. A Figura 2 apresenta o circuito do coletor, composto pela placa Heltec e pelos dois sensores de nível (ultrassônico e LIDAR). O circuito foi alimentado com 5V - 500mA e, para as ligações do sensor ultrassônico, foi necessário montar um divisor de tensão com os resistores de $5 k \Omega$ e $10 k \Omega$, para garantir que no terminal de leitura (echo) o sinal de tensão que indica que o sinal sonoro foi recebido chegasse em $3.3 \mathrm{~V}$ e não $5 \mathrm{~V}$, evitando possíveis danos ao sensor.

\footnotetext{
${ }^{7}$ https://shopee.com.br/

8 https://heltec.org/project/wifi-lora-32/

${ }^{9}$ http://bit.ly/semtech-sx1276
} 


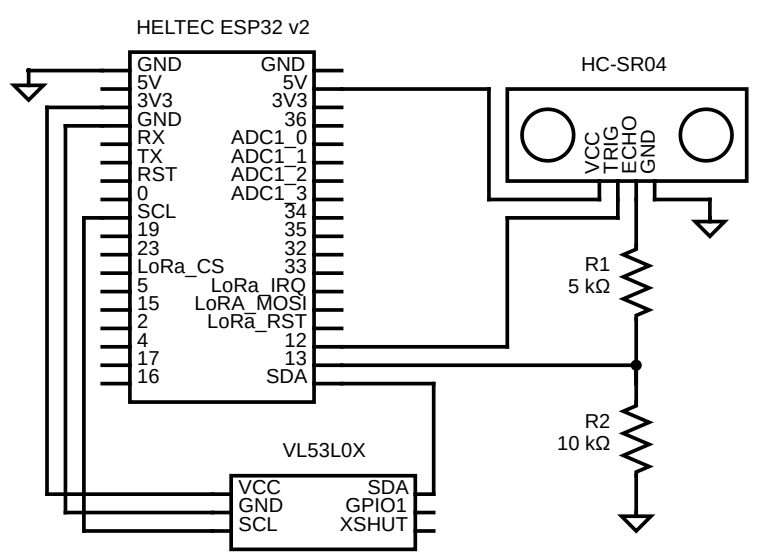

Figura 2. Circuito Esquemático do hardware utilizado no Coletor Inteligente.

Para esta etapa de avaliação de sensores de nível, a comunicação Serial/USB foi utilizada devido sua simplicidade. A lógica de funcionamento do firmware é apresentada no fluxograma da Figura 3. Primeiramente, a placa Heltec realiza a leitura de 20 amostras de valores de distância do sensor ultrassônico e LIDAR. Em seguida, são aplicados filtros (média e mediana) nos valores do sensor ultrassônico, com o objetivo de suavizar os picos de leituras. Após isso, a mensagem para envio é criada, sendo constituída dos valores brutos dos sensores e valores filtrados. Por fim, a mensagem é enviada via protocolo serial para o notebook a uma frequência de $50 \mathrm{~Hz}$. O Notebook utilizado nos experimentos foi o ASUS K46CA.309 v1.0, com processador i5-3317U e 8G RAM. É importante destacar que os valores do número de amostras consideradas para aplicação de filtros e a frequência de envio de dados foram escolhidos para fins de avaliar o desempenho dos sensores somente. Dessa forma, nas próximas etapas desta pesquisa, serão substituídos por valores que refletirão o cenário real da coleta de pilhas e baterias.

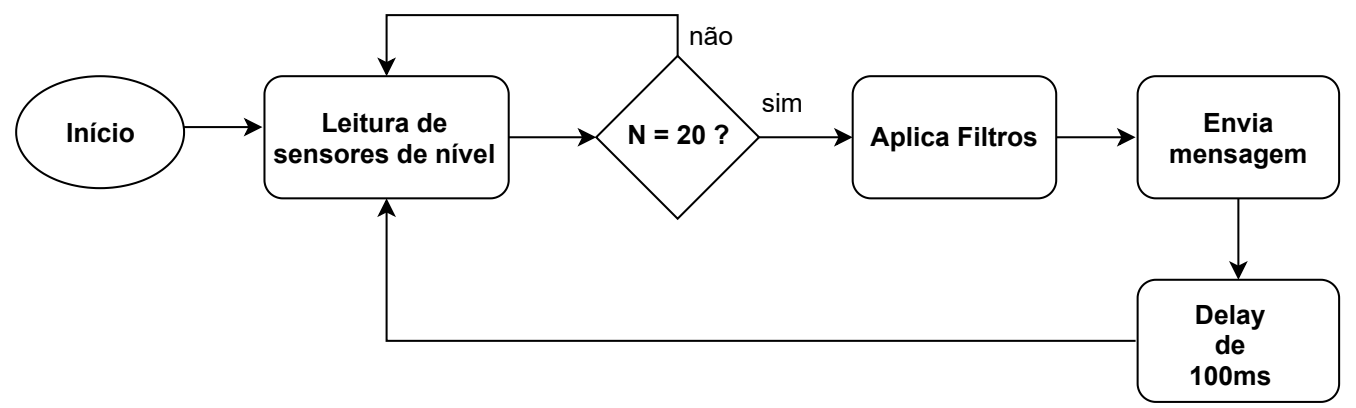

Figura 3. Lógica de monitoramento do nível do Coletor Inteligente.

\subsection{Configurações do experimento no Coletor Inteligente}

A duração do experimento foi de três dias (24 à 27/11/2020) e foram testadas duas situações de descarte de pilhas e baterias. No primeiro dia, o descarte foi realizado duas vezes, com duração de 10 minutos, para que se observasse o comportamento dos sensores em situações de grande quantidade de resíduos. Nos demais dias, os descartes foram 
feitos em períodos de tempo aleatórios e em pouca quantidade de pilhas (6 a 10 pilhas), configurando comportamento convencional de descarte.

A Figura 4(a) apresenta as medidas entre o protótipo de sensoriamento de nível, coletor e balde de resíduos. O balde, representado pelo cilindro, é responsável por armazenar as pilhas e baterias descartadas, atingindo capacidade total em $24 \mathrm{~cm}$. A posição do circuito de sensoriamento foi representada pelo losango, em azul, e sua distância até a parte inferior do coletor corresponde a $36 \mathrm{~cm}$. Entre o circuito e o topo do balde a distância é de $12 \mathrm{~cm}$. A variável $d$, que deverá ter valor diminuído durante o experimento, corresponde à distância que será retornada pelos sensores de nível. Para o início do experimento, o balde de resíduos foi previamente enchido em parte com areia, devido a limitação na quantidade pilhas e baterias disponíveis. Dessa forma, a medida inicial de enchimento que será detectada pelos sensores deve ser aproximadamente $22 \mathrm{~cm}$ e a final $12 \mathrm{~cm}$, valor que configura o enchimento do balde. As Figuras 4(b) e 4(c) mostram as condições iniciais e finais do coletor durante o experimento. A próxima seção apresenta os resultados obtidos por meio do sensoriamento de nível do coletor.

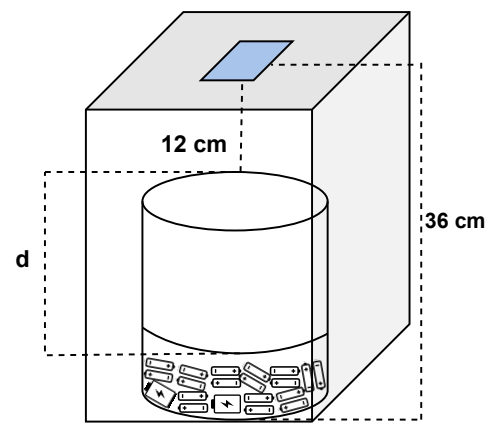

(a) Esquemático do Coletor.

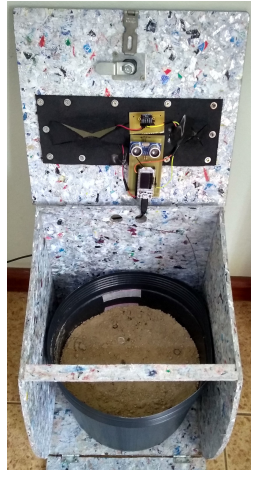

(b) Início do teste.

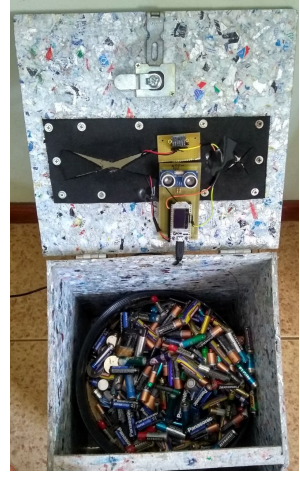

(c) Final do teste.

Figura 4. Ambiente de teste de sensoriamento do Coletor Inteligente.

\section{Discussão dos Resultados}

Esta seção tem como objetivo discutir os resultados obtidos por meio dos experimentos de sensoriamento de nível do coletor inteligente descritos na seção anterior, comparando os resultados dos sensores ultrassônico e LIDAR. Após análise dos dados coletados, foi possível criar o gráfico final de monitoramento (Figura 5), que consiste no nível de enchimento do balde de resíduos durante o período do teste. Ele compara o comportamento do sensor ultrassônico e LIDAR representados, respectivamente, pelas linhas azul e laranja. O gráfico ideal resultante esperado, em lilás, é próximo ao formato de uma escada decrescente, pois quanto maior a quantidade de resíduos, menor a distância medida esperada. Dentre os sensores, o LIDAR apresentou o comportamento mais próximo ao esperado.

No primeiro dia, pode-se observar duas regiões de picos nos gráficos, que correspondem ao descarte de resíduos por dez minutos, duas vezes durante o dia no intervalo de duas horas. Para este caso, ambos os sensores apresentaram comportamentos ruidosos durante o período de descarte, estabilizando em seguida. Entretanto, o ultrassônico apresenta picos que ultrapassam o próprio tamanho do balde de resíduos $(24 \mathrm{~cm})$, ao contrário do LIDAR que mesmo desestabilizado, teve leituras decrescentes. Para os demais dias 


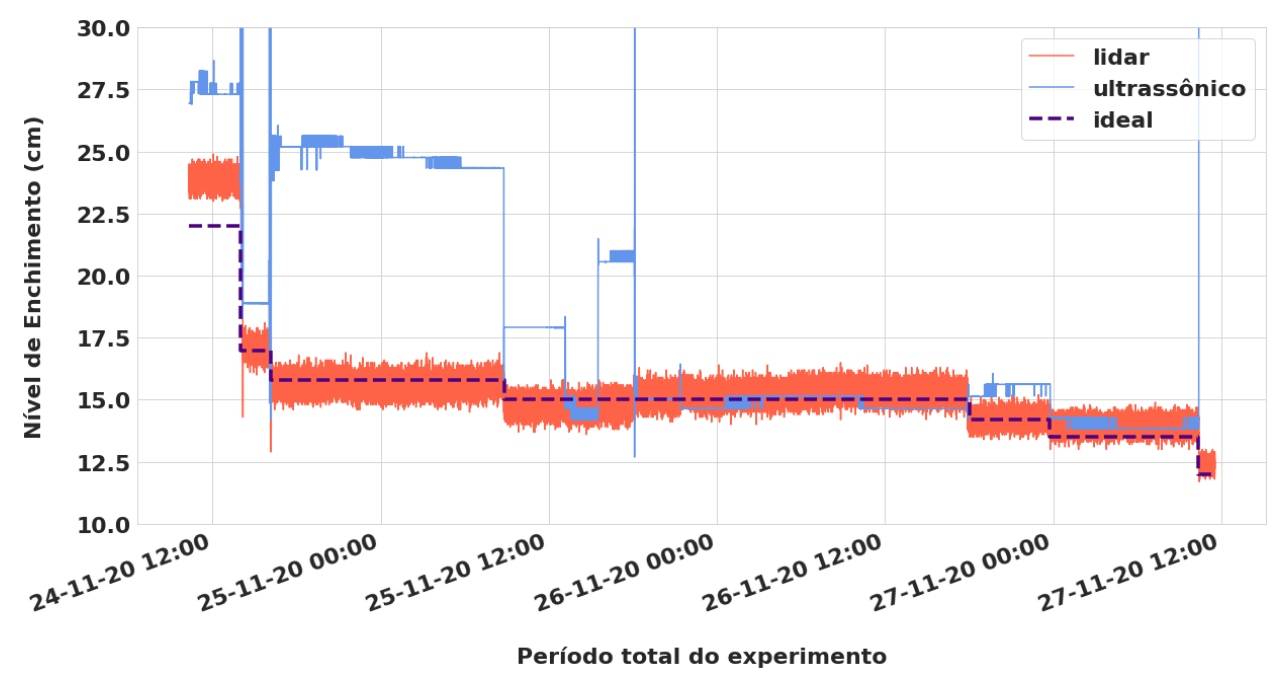

Figura 5. Comparativo entre sensor ultrassônico e LIDAR.

do experimento (25-27/11), em que o descarte era feito em momentos aleatórios do dia e em quantidade de 6-10 pilhas, pode-se perceber que as leituras do LIDAR se mantiveram estáveis em relação às do ultrassônico. No final do experimento, o ultrassônico não tinha conseguido estabilizar suas leituras, mesmo passadas duas horas do último descarte.

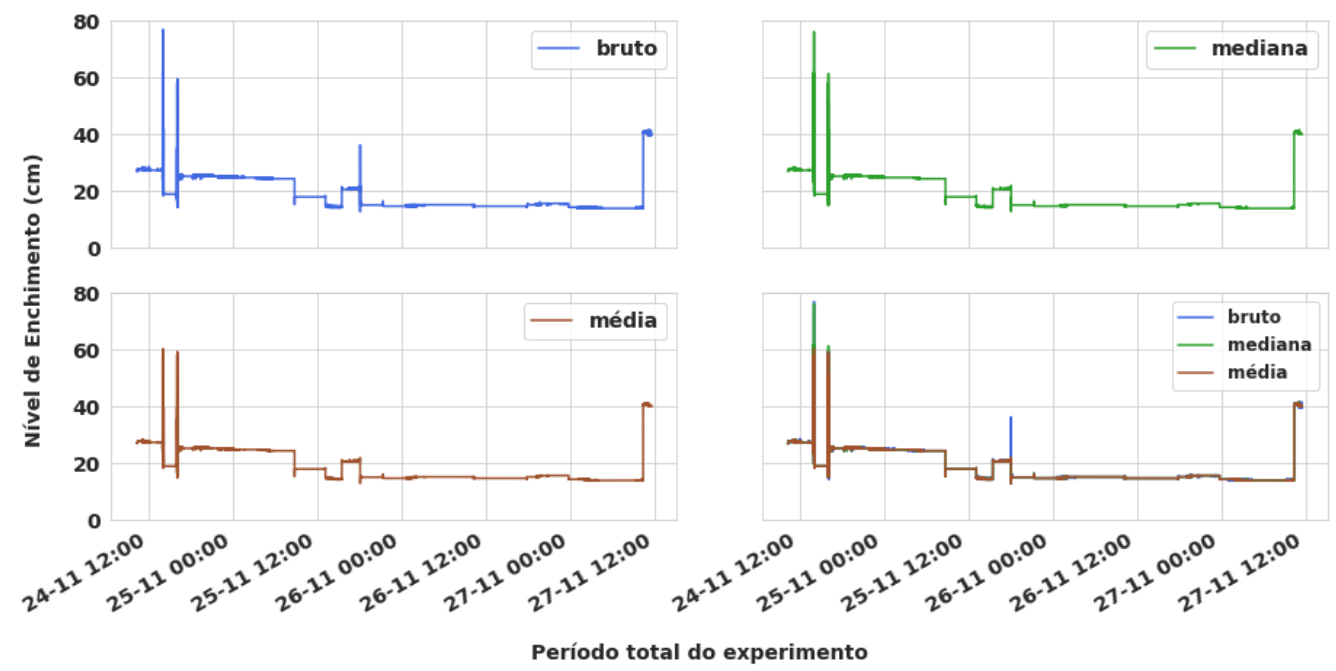

Figura 6. Filtros aplicados nas leituras do sensor ultrassônico.

Em relação aos filtros utilizados nas leituras do ultrassônico, a Figura 6 apresenta o valor bruto, valor filtrado com média, valor filtrado com mediana e todos eles sobrepostos, respectivamente. Observa-se que ambos os filtros conseguiram diminuir alguns picos de outliers, entretanto, os valores máximos de distância obtidos durante os experimentos foram $60.29 \mathrm{~cm}$ e $76.13 \mathrm{~cm}$, para média e mediana, respectivamente; que é acima do esperado para a faixa de leituras do experimento $(22-12 \mathrm{~cm})$. A Figura 7, ilustra o que pode estar ocorrendo para esta instabilidade nas leituras. Nela, o sensor ultrassônico transmite um novo sinal sonoro, em azul, que colidirá com o Objeto 1, e que deveria ser refletido de volta para que o mesmo calcule a distância entre ambos. Entretanto, com o desnível de altura ocasionado pelo enchimento de pilhas de baterias, o que pode 
estar acontecendo é que antes do sinal novo refletido chegar ao sensor, sinais antigos, em vermelho, refletidos por outros objetos mais distantes ou até mesmo pelas paredes do coletor, podem estar chegando primeiro, ocasionando os picos e consequentes erros nas leituras de distância [Morgan 2014]. Este comportamento também pode ser observado no trabalho de Baldo et al. [2021], cuja avaliação de desempenho deste sensor apresentou gráficos resultantes com picos e instabilidades durante o período de teste considerado.

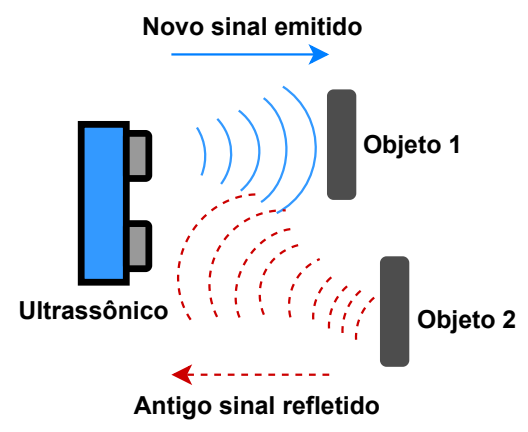

(a) Erro na leitura de distância.

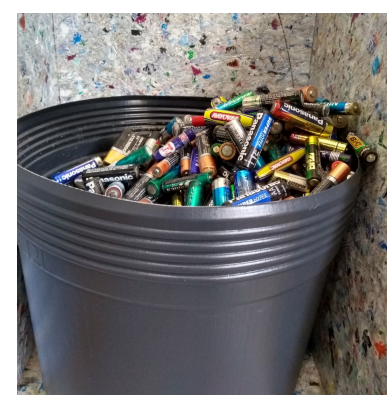

(b) Desnível de resíduos.

Figura 7. Cenário de instabilidade do sensor ultrassônico.

A Tabela 4 sumariza os resultados obtidos, apresentando os valores de erros absolutos para ultrassônico, filtros e LIDAR. As primeiras colunas de máximos e mínimos correspondem aos valores de picos durante o experimento para cada tipo de dado. As colunas finais representam os valores de erro absoluto para os valores máximos e mínimos do experimento, sendo calculados pela diferença entre o valor esperado e o medido. $\mathrm{O}$ valor máximo esperado para início e fim do experimento são 22 e $12 \mathrm{~cm}$, respectivamente. A tabela evidencia os valores incorretos das leituras do ultrassônico e, apesar dos mínimos absolutos representarem comportamento correto, os gráficos anteriores mostram que o sensor não foi capaz de estabilizar as leituras nessa faixa ao final do experimento. $\mathrm{O}$ LIDAR teve os melhores resultados sendo, portanto, o sensor mais indicado para a continuidade da pesquisa para o coletor de pilhas e baterias, pois apresentou resposta superior ao ultrassônico, tanto durante o descartes longos como em curtos períodos de tempo.

Tabela 4. Erros absolutos das distâncias obtidas pelo sensoriamento de nível.

\begin{tabular}{cccccc}
\hline Sensores & Tipo de dado & Máx. $(\mathbf{c m})$ & Mín. $(\mathbf{c m})$ & Máx. Abs. $( \pm \mathbf{c m})$ & Mín Abs. $( \pm \mathbf{c m})$ \\
\hline \hline \multirow{3}{*}{ Ultrassônico } & bruto & 77.00 & 12.69 & 55.00 & 0.69 \\
\cline { 2 - 6 } & média & 60.29 & 12.82 & 38.29 & 0.82 \\
\cline { 2 - 6 } & mediana & 76.13 & 12.80 & 54.13 & 0.80 \\
\hline \hline \multirow{2}{*}{ LIDAR } & bruto & 24.9 & 11.70 & $\mathbf{2 . 9}$ & $\mathbf{0 . 3}$ \\
\hline
\end{tabular}

\section{Considerações Finais}

Este trabalho propôs uma plataforma inteligente e sustentável para coleta de resíduos, baseada em IoT e LPWAN, com o objetivo de economizar recursos (e.g., combustível, 
mão de obra) e gerar indicadores de sustentabilidade. O foco foi definir o sensor de monitoramento de nível dos coletores inteligentes, que corresponde ao primeiro estágio da plataforma. Foram realizados testes e análises comparativas entre os sensores ultrassônico e LIDAR, permitindo concluir que o LIDAR é o mais apropriado para o coletor de pilhas e baterias, utilizado como caso de estudo, pois demonstrou estabilidade durante a maior parte do experimento e teve erro absoluto máximo de apenas $\pm 2.9 \mathrm{~cm}$, mostrando-se superior ao ultrassônico que teve máximo absoluto de $\pm 55 \mathrm{~cm}$. Outra possibilidade de estudo para a fase de monitoramento seria analisar a combinação de sensores para determinar o nível de enchimento dos coletores, entretanto para esta solução esta alternativa não é interessante pois implica no aumento do custo e do consumo do hardware.

Os indicadores de sustentabilidade propostos para serem gerados pela plataforma são baseados nos ODSs da ONU e giram em torno dos objetivos referentes à cidades sustentáveis, consumo e produção responsáveis e ação contra mudanças climáticas. $\mathrm{O}$ próximo passo será implementá-los em um sistema web, possibilitando que sejam monitorados pelos órgãos responsáveis bem como consultados publicamente. Outros componentes da plataforma também serão avaliados no futuro, como a infraestrutura de rede LoRaWAN. Espera-se que, quando todas estas etapas forem validadas, novos tipos de resíduos possam ser coletados por meio da infraestrutura desenvolvida, com adequações de hardware (e.g., tipo de sensor) e no conteúdo do pacote de dados transmitidos pelos coletores inteligentes, que depende do tipo de resíduo (e.g., hospitalar, químico).

\section{Agradecimentos}

A realização deste estudo foi possível graças ao apoio financeiro e suporte técnico dos parceiros na Prefeitura da Unicamp, Smart Campus Unicamp e Konker.

\section{Referências}

Ali, T., Irfan, M., Alwadie, A. S., and Glowacz, A. (2020). Iot-based smart waste bin monitoring and municipal solid waste management system for smart cities. AJSE, 45:10185-10198.

Anagnostopoulos, T., Zaslavsky, A., Kolomvatsos, K., Medvedev, A., Amirian, P., Morley, J., and Hadjieftymiades, S. (2017). Challenges and opportunities of waste management in iot-enabled smart cities: a survey. IEEE T-SUSC, 2(3):275-289.

Anh Khoa, T., Phuc, C. H., Lam, P. D., Nhu, L. M. B., Trong, N. M., Phuong, N. T. H., Dung, N. V., Tan-Y, N., Nguyen, H. N., and Duc, D. N. M. (2020). Waste management system using iot-based machine learning in university. IWCMC, 2020.

Arducore (2021). Sensor Infravermelho Reflexivo Industrial E18-d80nk. http://bit. ly/arducore-infravermelho.

Bakhshi, T. and Ahmed, M. (2018). Iot enabled smart city waste management using machine learning analytics. In 2018 2nd ICECE, pages 66-71. IEEE.

Baldo, D., Mecocci, A., Parrino, S., Peruzzi, G., and Pozzebon, A. (2021). A multi-layer lorawan infrastructure for smart waste management. Sensors, 21(8):2600.

Bertoleti, P. (2017). Medindo vazão utilizando um sensor ultra-sônico de distância. http: / / bit. ly/embarcados-ultrassonico.

Cruz, N., Cota, N., and Tremoceiro, J. (2021). Lorawan and urban waste management-a trial. Sensors, 21(6):2142. 
ECAM (2021). O que é a Agenda 2030 e quais os seus objetivos. https://bit. Iy/ ods-a2030.

ETT (2021). E18-D80NK-N Datasheet. https://bit.ly/ir-d80nk.

Fatimah, Y. A., Govindan, K., Murniningsih, R., and Setiawan, A. (2020). Industry 4.0 based sustainable circular economy approach for smart waste management system to achieve sustainable development goals: A case study of indonesia. J. clean prod., 269:122263.

Fedchenkov, P., Zaslavsky, A., Medvedev, A., Anagnostopoulos, T., Sosunova, I., and Sadov, O. (2017). Supporting data communications in iot-enabled waste management. In Internet of Things, Smart Spaces, and Next Generation Networks and Systems, pages 163-174. Springer.

Ferreira, W. (2020). Lidar - ToF. http://bit. ly/embarcados-lidar-tof.

Hatta, T. E. and Borin, J. F. (2018). Dispositivo iot para coleta inteligente de pilhas e baterias. Relatório Técnico - IC-PFG-18-12.

Hussain, A., Draz, U., Ali, T., Tariq, S., Irfan, M., Glowacz, A., Antonino Daviu, J. A., Yasin, S., and Rahman, S. (2020). Waste management and prediction of air pollutants using iot and machine learning approach. Energies, 13(15):3930.

Lozano, Á., Caridad, J., De Paz, J., Villarrubia González, G., and Bajo, J. (2018). Smart waste collection system with low consumption lorawan nodes and route optimization. Sensors, 18(5):1465.

Marques, P., Manfroi, D., Deitos, E., Cegoni, J., Castilhos, R., Rochol, J., Pignaton, E., and Kunst, R. (2019). An iot-based smart cities infrastructure architecture applied to a waste management scenario. Ad Hoc Networks, 87:200-208.

Melakessou, F., Kugener, P., Alnaffakh, N., Faye, S., and Khadraoui, D. (2020). Heterogeneous sensing data analysis for commercial waste collection. Sensors, 20(4):978.

Morgan, E. J. (2014). Hc-sr04 ultrasonic sensor.

Pardini, K., Rodrigues, J., Kozlov, S., Kumar, N., and Furtado, V. (2019). Iot-based solid waste management solutions: A survey. JSAN, 8(1):5.

Pereira, V. (2017). Conheça a tecnologia LoRa e o protocolo LoRaWAN (LOR001). http://bit.ly/lora-lorawan.

Prefeitura Universitária, U. (2016). Programa de coleta de pilhas e baterias - Instalação de coletores. http://bit.ly/unicamp-coletores.

Quadros, D. (2021). Aprenda a construir uma balança com Arduino e módulo HX711. http://bit.ly/filipepflop-hx711.

SDSN (2021). Global Indicators for the SDGs. https: //indicators.report/.

Sheng, T. J., Islam, M. S., Misran, N., Baharuddin, M. H., Arshad, H., Islam, M. R., Chowdhury, M. E., Rmili, H., and Islam, M. T. (2020). An internet of things based smart waste management system using lora and tensorflow deep learning model. IEEE Access, 8:148793-148811.

Shyam, G. K., Manvi, S. S., and Bharti, P. (2017). Smart waste management using internet-of-things (iot). In 2017 2nd ICCCT, pages 199-203. IEEE.

Sohag, M. U. and Podder, A. K. (2020). Smart garbage management system for a sustainable urban life: An iot based application. Internet of Things, 11:100255.

STMicroelectronics (2016). V15310x - world smallest time-of-flight rangingfeatures. In Datasheet DocID029104 Rev 1. STM.

Straub, M. (2019). Balança Arduino com Célula de Carga e HX711. http://bit.ıy/ usinainfo-load-cell.

TeraBee (2020). Time of Flight principle. http://bit.ly/tof-principle. 